\title{
Towards a full understanding of water splitting in photosynthesis
}

\author{
James Barber \\ Wolfson Laboratories, Biochemistry Building, Department of Biological Sciences, \\ Imperial College London, South Kensington Campus, London SW7 2AZ, UK
}

\begin{abstract}
The capture and conversion of solar radiation by photosynthetic organisms directly or indirectly provides energy for almost all life on our planet. About 2.5 billion years ago a remarkable biological "machine" evolved known as photosystem two (PSII). This machine can use the energy of visible light (actually red quanta of $\sim 1.8 \mathrm{eV}$ ) to split water into dioxygen and "hydrogen". The latter is made available as reducing equivalents, ultimately destined to convert carbon dioxide to organic molecules. In PSII, the "hydrogen" reduces plastoquinone $(\mathrm{PQ})$ to plastoquinol $\left(\mathrm{PQH}_{2}\right)$. The water splitting process takes place at a catalytic centre composed of $4 \mathrm{Mn}$ atoms and the reactions involved are chemically and thermodynamically challenging. The process is driven by a photooxidised chlorophyll molecule $\left(\mathrm{P}_{680^{\circ}}{ }^{+}\right)$and involves electron/proton transfer reactions aided by a redox active tyrosine residue situated between the $4 \mathrm{Mn}$ cluster and P680. The $\mathrm{P}_{680^{\bullet+}}$ species is generated by light induced rapid electron transfer (a few picoseconds) to a primary acceptor, pheophytin $a$, before being transferred to PQ acceptors. Electron and x-ray crystallographic studies are now starting to reveal the structural basis for these reactions including the light harvesting processes. The $4 \mathrm{Mn}$ atom-cluster has been visualised as have the chlorophylls that constitute P680. The scene is now set to fully elucidate the reactions of PSII and possibly mimic them in an artificial photochemical system that could split water and produce hydrogen.
\end{abstract}

\section{INTRODUCTION}

Water is the source of reducing equivalents for almost all life on our planet. The process of photosynthesis uses solar energy to split water into oxygen and "hydrogen". While the former is released from the photosynthetic organism as dioxygen, the latter is used to reduce carbon dioxide to organic molecules. The reduction process involves a complex set of proton/electron transfers followed by the interplay of enzyme controlled biosynthetic pathways. Dioxygen is therefore the by-product of water splitting and provides us not only with our aerobic atmosphere but its continuous release from photosynthetic organisms also maintains the ozone layer that protects terrestrial life from ultraviolet radiation. The "invention" of the water splitting process about 2.5 billion years ago gave rise to an explosion of life on our planet since biology had found the ultimate source of reducing equivalents which was available in unlimited amounts. In fact there is about $1.37 \times 10^{9}$ GTonnes of water on our planet today [1]. Photosynthetic activity results in the release of 260 GTonnes of oxygen into the atmosphere annually and the storing of 100 GTonnes of carbon in the form of biomass [2].

The water splitting reaction takes place in a chlorophyll-binding protein complex embedded in the lipid bilayer of the photosynthetic thylakoid membrane. It is composed of several subunits and is known as Photosystem II (PSII). There is little doubt that PSII evolved from a simpler photosystem akin to those found in purple photosynthetic bacteria to day which oxidise "hydrogen" donors like $\mathrm{H}_{2} \mathrm{~S}$ and organic acid. The evolution of PSII, however, was surely the "Big Bang of Evolution" since it made it possible for plant and animal life to prosper on an enormous scale.

\section{PHOTOSYSTEM II}

This photosystem uses solar energy to oxidise water and reduce plastoquinone (PQ)

$$
2 \mathrm{H}_{2} \mathrm{O}+2 \mathrm{PQ} \stackrel{4 h v}{\longrightarrow} 2 \mathrm{PQH}_{2}+\mathrm{O}_{2}
$$

The reduced plastoquinone $\left(\mathrm{PQH}_{2}\right)$ is the starting point for the transfer of reducing equivalents to $\mathrm{CO}_{2}$ and involves one further input of solar energy via charge transfer in photosystem I (PSI). It is found in all forms of plants, algae and oxyphotobacteria and consists of a reaction centre (RC) core with an associated light harvesting (LH) system. Although the RC core is essentially identical in all types of oxygenic organisms the LH systems can vary considerably. In plants and green algae, the LH systems contain chlorophyll $a$, chlorophyll $b$ and carotenoids. However, red algae and cyanobacteria do not contain chlorophyll $b$ but employ phycobilins as LH pigments. Some other organisms, such as brown algae, diatoms etc. contain other forms of chlorophyll, such as chlorophyll $c$ and chlorophyll $d$. In contrast to the LH systems, the PSII RC core contains only chlorophyll $a$ with one known exception, that being the oxyphotobacterium Acaryochloris marina which contains chlorophyll $d$ as well as chlorophyll $a$ in its PSII RC core [3]. However, as far as we know, in 


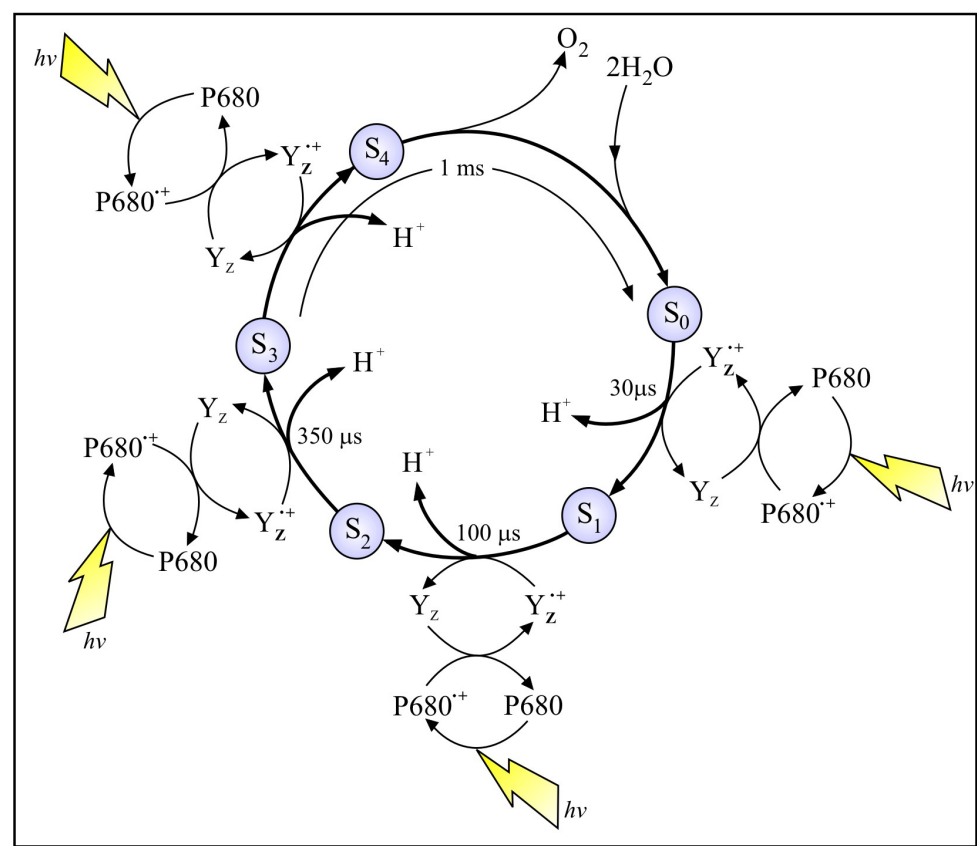

Figure 1. The S-state cycle for the oxygen evolution reaction, as first suggested by Kok et al. [46], modified to accommodate the model proposed by Babcock and colleagues (reviewed in [19, 20]). The scheme is based on the fact that, when a photosynthetic organism is exposed to a series of saturating flashes of light, the evolution of oxygen follows a period of four. This indicates that four oxidising equivalents must accumulate at a single catalytic centre before a dioxygen molecule is formed and released. Therefore, there must be four light-induced redox turnovers of PSII reaction centre chlorophylls (P680) and tyrosine $\left(\mathrm{Y}_{\mathrm{Z}}\right)$ to produce the four oxidising equivalents accumulated in the Mn cluster. Dioxygen is produced on the $\mathrm{S}_{4}$ to $\mathrm{S}_{0}$ transition. According to the hypothesis of Babcock and colleagues, each photoinduced step of the S-state cycle $\left(\mathrm{S}_{0}\right.$ through to $\left.\mathrm{S}_{4}\right)$ involves the concerted removal of an electron and a proton from two bound water molecules. Other proposed mechanisms do not invoke a hydrogen abstraction at each step of the cycle [21, 22].

all cases the species involved in primary charge separation, P680, is chlorophyll $a$. When excited P680 donates an electron to pheophytin (Pheo) to form the radical pair $\mathrm{P}_{680^{\circ}} \mathrm{Pheo}^{\circ-}$ in a few picoseconds [4]. The electron is then rapidly transferred to a bound $P Q$ molecule known as $\mathrm{Q}_{A}$ and then to $\mathrm{Q}_{B}$ which is also a PQ molecule [5]. However, $\mathrm{Q}_{\mathrm{B}}$, unlike $\mathrm{Q}_{\mathrm{A}}$, is able to undergo a full reduction by a two-electron/proton transfer and the resulting $\mathrm{PQH}_{2}$ is released from PSII into the lipid matrix of the membrane.

The $\mathrm{P} 680^{\circ}+$ species is highly oxidising with an estimated potential of $1.1 \mathrm{eV}$ or more. Such a high redox potential is required to oxidise water. Recent electron [6-9] and x-ray crystallography [10, 11] suggests that the P680 excited state $\left(\mathrm{P} 60^{\star}\right)$ is shared between 4 chlorophyll molecules spaced about $10 \AA$ from each other (centre-to-centre). It is also possible that the initially formed $\mathrm{P} 60^{\circ+}$ is delocalised over all or some of these chlorophylls which means that they are all high oxidising species [12, 13]. However it seems that the $\mathrm{P} 680^{\circ}+$ oxidising equivalent is rapidly localised on a specific chlorophyll which is close to a tyrosine residue $\left(\mathrm{Y}_{\mathrm{Z}}\right)$ [14]. This results in the oxidation of the $\mathrm{Y}_{\mathrm{Z}}$ to $\mathrm{Y}_{\mathrm{Z}}^{\bullet+}$ and the proba- ble loss of a proton from its phenol group leads to the neutral tyrosine radical $\left(\mathrm{Y}_{\mathrm{Z}}^{\bullet}\right)[15]$.

The splitting of water occurs at a catalytic centre that consists of four manganese atoms. The Mn-cluster binds two water substrate molecules that require four oxidising equivalents to produce dioxygen. Thus four photochemical turnovers must occur sequentially before $\mathrm{O}_{2}$ is evolved as shown in the S-state cycle in Figure 1 .

\section{MECHANISM OF WATER OXIDATION}

The electron transfer reactions that occur on the reducing side of PSII and bring about the reduction of plastoquinone to plastoquinol are remarkably similar to those occurring in the RCs of purple photosynthetic bacteria [16]. It is, however, the oxidising side that makes PSII so unique. The high redox potential of $\mathrm{P} 680^{\bullet+}$ is required to oxidise water and the four oxidising equivalents generated by the four photochemical turnovers of the S-state cycle (see Figure 1) are localised on or near the $(\mathrm{Mn})_{4}$-cluster. There is increasing evidence to suggest that these four oxidising equivalents do not accumulate but are utilized throughout the S-state cy- 
cle to extract electrons and protons from two substrate water molecules bound to the $(\mathrm{Mn})_{4}$-cluster at the $\mathrm{S}_{0}$ state. Moreover it has been known for some time that calcium and chloride ions are required for water oxidation catalysis and are believed to be located close to the $(\mathrm{Mn})_{4}$-cluster $[17,18]$. One possible mechanism for the subsequent oxidation processes involves the concerted removal of a proton and electron from the two substrate water molecules at each step of the S-state cycle. This hydrogen abstraction model was proposed by Babcock, Tommos and co-workers [19, 20] and is shown in Figure 2A . In this scheme the deprotonation of $\mathrm{Y}_{\mathrm{Z}}^{+}$ to create a neutral radical $Y_{Z}^{\bullet}$ facilitates its subsequent reduction by accepting an electron and proton on each step of the S-state cycle. The radical states formed on the substrate water intermediates are stabilised by the increase in the oxidation level of the $(\mathrm{Mn})_{4}$-cluster. As a proton, as well as an electron, is ejected at each step of the S-state cycle the increase in the oxidation of the $(\mathrm{Mn})_{4}$-cluster does not impose any coulombic restrictions because the system remains electroneutral. However there is experimental evidence to suggest that this electroneutral scheme does not happen and that the $S_{1}$ $\mathrm{S}_{2}$ transition involves only electron transfer from the $(\mathrm{Mn})_{4}$-cluster. With this in mind alternative schemes to describe water oxidation have been suggested. For example, Brudvig and colleagues [21, 22] have proposed the scheme shown in Figure 2B. Again it is believed that the two substrate water molecules are bound at the $\mathrm{S}_{0^{-}}$ state with one associated with $\mathrm{Ca}^{2+}$. The mechanism requires that during the S-state cycle one of the Mn atoms becomes highly oxidised to $\mathrm{Mn}(\mathrm{V})$ and a $\mathrm{Ca}^{2+}$ acts as a Lewis acid by facilitating a nucleophilic attack on an electron deficient $\mathrm{Mn}(\mathrm{V})=\mathrm{O}$ intermediate in order to allow the O-O bond to form. Hydrogen atom abstraction is proposed for all steps except for the $S_{1}-S_{2}$ transition.

The two schemes shown in Figure 2 are reliant on $\mathrm{Y}_{\mathrm{Z}}^{\bullet}$ (i.e., $\mathrm{Y}_{\mathrm{Z}} \mathrm{O}^{\bullet}$ ) to act as $\mathrm{H}^{+}$as well as an electron acceptor. In the absence of a high-resolution structural model it has been proposed that D1-His190 is hydrogen bonded to $\mathrm{Y}_{\mathrm{Z}}$ and that the phenolic proton of tyrosine is transferred to this histidine. In turn this histidine transfers each proton generated by the water oxidation process to a second acceptor and a domino deprotonation occurs, eventually giving rise to proton release into the lumen. Here again, the measured proton release pattern does not seem to agree with Babcock's electroneutral theory for water oxidation and has been measured to be $1,0,1,2$ for the $S_{0}$ through to the $S_{4}$ state of the cycle by Junge and colleagues [23, 24]. However, because the catalytic site for water oxidation is buried within the large protein mass attached to the lumenal membrane surface, it is difficult to be sure if this stoichiometry is a true reflection of the proton release pattern from the two substrate water molecules.

In order to obtain a more reliable chemical mechanism for water oxidation, structural information is re- quired at better resolution than currently available. Ideally structures need to be obtained for each S-state of the $\mathrm{S}_{0}$ to $\mathrm{S}_{4}$ cycle and at a resolution better than $2.5 \AA$. Nevertheless, as described below, high-resolution electron microscopy and x-ray crystallography have paved the way towards this goal.

\section{STRUCTURE OF THE PSII RC CORE}

Very recently electron and x-ray crystallographic studies have provided information about the structure of the PSII RC core. This complex is made up of four chlorophyll binding proteins; D1, D2, CP43 and CP47 and a number of other proteins as depicted in Figure 3. The D1 and D2 proteins are very similar and bind all the redox active cofactor giving rise to water splitting and PQ reduction. CP43 and CP47 are also similar proteins whose main function seems to be light harvesting, with CP43 binding 13 chlorophyll $a$ molecules and CP47 binding $14[10,11]$. The five transmembrane helices of the D1 protein and the six transmembrane helices of CP43 are related to the corresponding helices of D2 and CP47 by a pseudo-2-fold axis (see Figure 4). In addition to these four subunits (which provide 22 transmembrane helices) there are 12 [9] or $14[10,11]$ additional single transmembrane subunits of which two PsbE and PsbF bind the haem of cytochrome b559.

As indicated in Figure 5, the four chlorophylls of $\mathrm{P} 680\left(\mathrm{P}_{\mathrm{D} 1}, \mathrm{P}_{\mathrm{D} 2}, \mathrm{Chl}_{\mathrm{D} 1}, \mathrm{Chl}_{\mathrm{D} 2}\right)$ are bound symmetrically to the $\mathrm{D} 1$ and $\mathrm{D} 2$ proteins that also bind two symmetry related Pheo molecules (only $\mathrm{Pheo}_{\mathrm{D} 1}$ is active) and PQ molecules ( $\mathrm{Q}_{\mathrm{A}}$ on the $\mathrm{D} 2$ side, $\mathrm{Q}_{\mathrm{B}}$ on the $\mathrm{D} 1$ side) (see Figure 5 ). $\mathrm{Y}_{\mathrm{Z}}$ is tyrosine 161 of the $\mathrm{D} 1$ protein while a symmetrically related tyrosine $\left(\mathrm{Y}_{\mathrm{D}}\right)$ on the $\mathrm{D} 2$ protein is not directly involved in water oxidation although it is oxidise by $\mathrm{P} 680^{\bullet+}$.

The Mn-cluster is bound only on the D1 side and therefore breaks the pseudo-2-fold symmetry (see Figure 6). Based on site directed mutagenesis [25] and recent x-ray analyses [10,11] possible ligands are located at the C-terminus of this protein and, as shown in Figure 6 , in the region of its CD lumenal surface helix. The Mn-cluster is stabilised by the extrinsic $33 \mathrm{kDa}$ PsbO protein which also seems to provide an environment for maintaining the $\mathrm{Ca}^{2+}$ and $\mathrm{Cl}^{-}$cofactors [26]. In plants and green algae two other extrinsic proteins $\mathrm{PbsP}$ and PsbQ associate with the PsbO protein. The PsbU and PsbV proteins in red algae and oxyphotobacteria replace these. Their functions are unknown but seem to form a diffusion barrier around the labile $4 \mathrm{Mn}$ cluster. Interestingly the PsbV protein is a c-type cytochrome (Cyt C550) but does not seem to be redox active during normal PSII functional activity.

The resolutions of the x-ray maps $(\sim 3.7 \AA)$ are, at present, not sufficient to give a precise structure for the $4 \mathrm{Mn}$-atom cluster but is seems that three Mn atoms are positioned at the corners of an isosceles triangle (see 


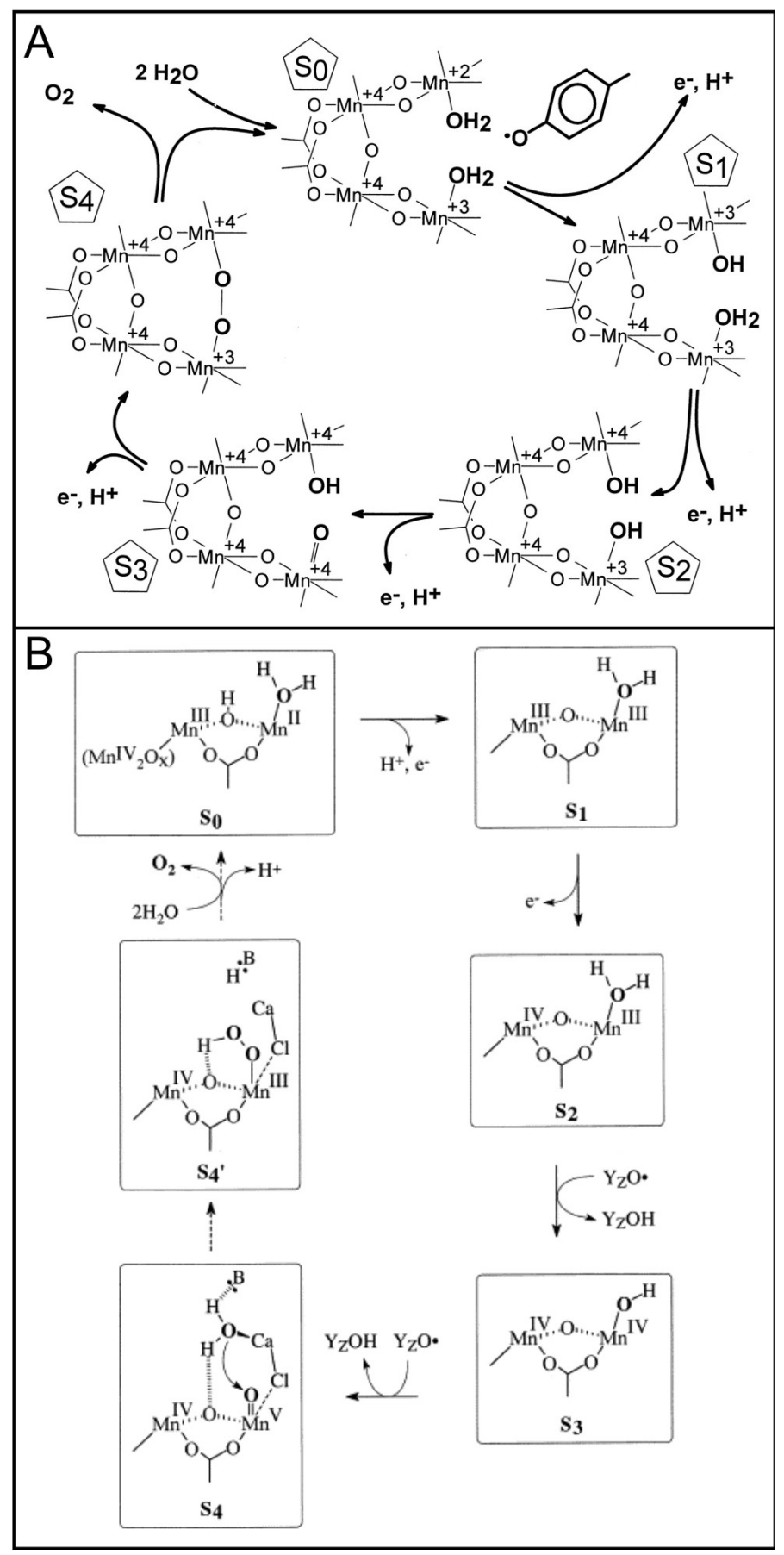

Figure 2. Two mechanistic reaction schemes proposed for $\mathrm{H}_{2} \mathrm{O}$ oxidation. (a) The "hydrogen" abstraction scheme of Babcock and colleagues taken from Hoganson and Babcock [15]. (b) The "high valency" mechanism of Brudvig and colleagues taken from Vrettos et al. [21]. Although the Babcock scheme assumed a "dimer of dimer" model for the (Mn) 4 -cluster it is not dependent on this particular configuration and can be accommodated, according to recent X-ray models ([10, 11] and see Figure 6). The recent $x$-ray data also does not negate the Brudvig model but note that in this scheme only two Mn atoms are involved in the water oxidation chemistry. For clarity the two $\mathrm{Mn}$ atoms which do not undergo redox changes during $S$-state advancement (assumed to be $\mathrm{Mn}(\mathrm{IV})_{2} \mathrm{O}_{\mathrm{x}}$ ) are omitted beyond $\mathrm{S}_{0}$.

Figure 6) with the fourth Mn atom centrally located, either in (10) or slightly out (11) of plane. The distances between the proposed $\mathrm{Mn}$ atoms are consistent with the existence of di-oxo bridges (2.8 §) and in agreement 


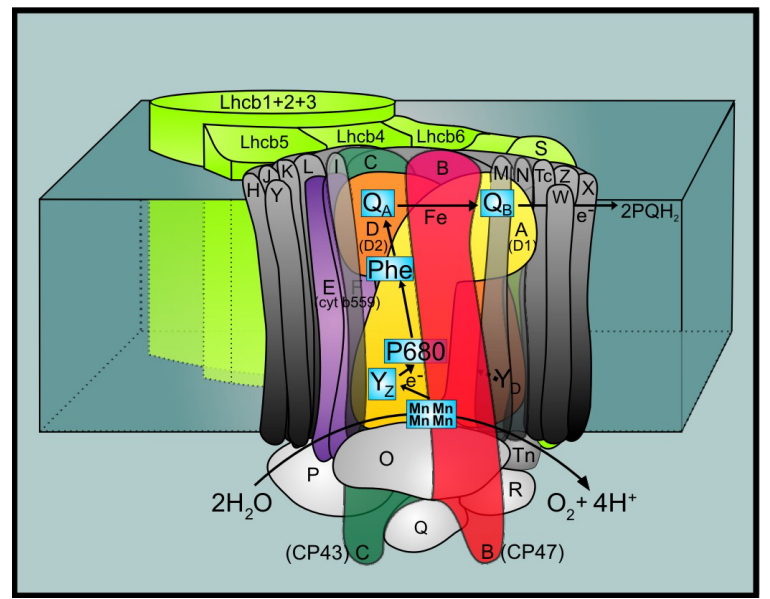

Figure 3. Cartoon of the structure and subunit composition of PSII of higher plants and green algae. The intrinsic and extrinsic proteins are labelled according to the gene nomenclature (e.g., $\mathrm{psb \textrm {A }}=\mathrm{A}$ ) with common designations given for the major subunits (e.g., $\mathrm{A}=\mathrm{D} 1$ protein). The outer light-harvesting proteins are coloured light green (intrinsic Lhcb/Cab proteins). The electron transfer pathway from water oxidation to plastoquinone reduction $\left(\mathrm{PQH}_{2}\right)$ is shown (see text).

with predictions from a range of spectroscopic techniques [27-29]. The poor quality of the $\mathrm{x}$-ray map does not allow visualisation of the $\mathrm{Ca}^{2+}$ or $\mathrm{Cl}^{-}$cofactors or of the water substrate molecules. However, assignments of density to tyrosine D1 Tyr $161\left(\mathrm{Y}_{\mathrm{Z}}\right)$ and possibly to histidine D1 His190 support the general concept that electron/proton transfer can occur from the water substrate molecules to $\mathrm{Y}_{\mathrm{Z}}^{\bullet}$ and that proton transfer from $\mathrm{Y}_{\mathrm{Z}}^{\bullet+}$ to a nearby base provides the neutral tyrosine radical required for hydrogen atom abstraction.

Over the years many studies, especially those involving resonance spectroscopy have provided information about the valency states of the Mn atoms in the cluster for different $\mathrm{S}$-states and about the interactions of cofactors $\left(\mathrm{Ca}^{2+}, \mathrm{Cl}^{-}\right)$and substrates $\left(\mathrm{H}_{2} \mathrm{O}, \mathrm{NH}_{3}\right.$, $\mathrm{NH}_{2} \mathrm{OH}$ ) which are reviewed in recent publications (see [30]). These have been rewarding studies but inevitably we will also need high-resolution structural data which can follow the fate of the two substrate water molecules during the S-state cycle.

\section{THERMODYNAMICS AND TOXICITY}

The oxidation of water is thermodynamically demanding. The average redox potential necessary to oxidise water to dioxygen is about $0.81 \mathrm{eV}$ at $\mathrm{pH} 7.0$ assuming a four electron process. According to electrochemical considerations higher redox potentials may be required for some steps. For example, as shown in Figure 7 , the redox potential of the individual oxidations

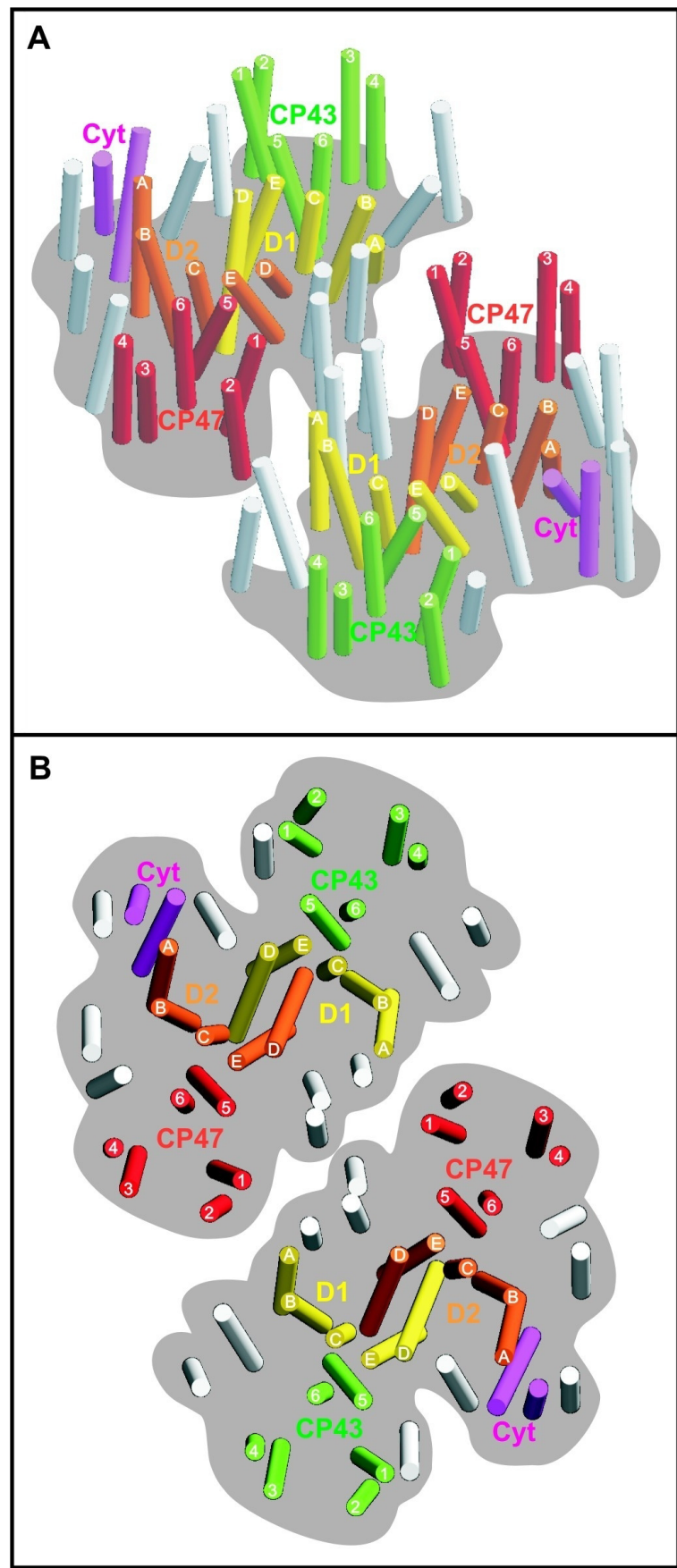

Figure 4. 3D structural model of PSII core dimer of spinach derived by electron crystallography [9, 47] showing (A) oblique view and (B) top view from the lumen. Colour coding of major subunits corresponds with that used in Figure 3.

proceeding via $\mathrm{H}_{2} \mathrm{O}_{2}$ or an $\mathrm{O}$-atom vary widely. For the $\mathrm{H}_{2} \mathrm{O}_{2}$ route (solid line) the redox potentials vary from $-0.45 \mathrm{~V}$ to $+2.33 \mathrm{~V}$ while in the pathway via an O-atom (solid and dashed lines) there are two large positive steps and two large negative steps. The minimum energy pathway $(+0.81 \mathrm{~V}$ per step) is shown by the 


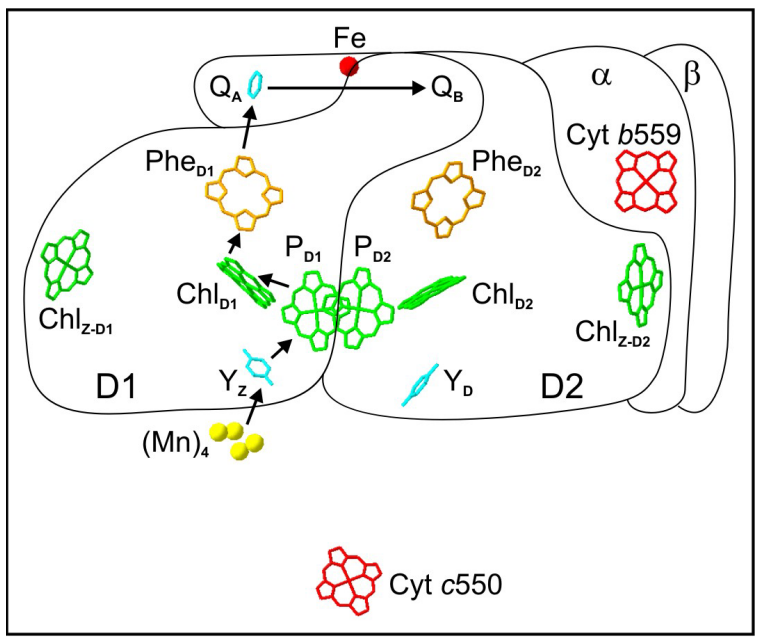

Figure 5. Organisation of the RC cofactors of PSII, derived by X-ray crystallography at 3.8-3.7 $\AA$ resolution $[10,11]$. The arrows show the direction of electron transfer from the $(\mathrm{Mn})_{4}$ cluster to the terminal plastoquinone acceptor $\mathrm{Q}_{\mathrm{B}}$. This quinone was not present in the 3D crystals analysed, but its position is inferred from the similarity between the structures of PSISI and the purple bacterial RCs [16]. An outline of the $\mathrm{D}_{1}, \mathrm{D}_{2}, \alpha$ - and $\beta$-subunits has been drawn as a cartoon to emphasise each protein-cofactor relationship in a diagrammatic manner. Also shown are the haems of cytochrome b559 and cytochrome c550 (PsbV protein).

dotted line. Therefore the estimated oxidising potential of $\mathrm{P} 680^{\circ+}$ of $1.1 \mathrm{eV}$ is barely enough and implies that photosynthetic water oxidation proceeds by a mechanism where each redox event is optimised to about $1 \mathrm{eV}$. Even so redox potential as high as $1 \mathrm{eV}$ brings problems. If the lifetime of $\mathrm{P} 680^{\circ+}$ is extended because water oxidation is inhibited for some reason, then there is the possibility that it will oxidise its local protein environment. Indeed, there is evidence that this occurs leading to irreversible damage of PSII by so called "donor side photoinhibition" [31]. Perhaps more serious is that the high oxidising potential of $\mathrm{P} 680^{\circ}+$ means that the P680 chlorophylls cannot use a carotenoid to quench any triplet states formed [32, 33]. This is because triplet transfer requires that the carotenoid is very close to the P680 chlorophyll (van der Waal distance) but if this were so then it would compete with water as a reductant for $\mathrm{P} 680^{\circ}$. This is the only example in photosynthesis where carotenoids are unable to carry out this protective role. Instead the $\mathrm{P} 680$ triplets $\left(\mathrm{P} 60^{3}\right)$ interact with dioxygen which is normally in its triplet state $\left(\mathrm{O}_{2}{ }^{3}\right)$ to form highly reactive singlet oxygen $\left(\mathrm{O}_{2}{ }^{1}\right)$

$$
\mathrm{P} 680^{3}+\mathrm{O}_{2}{ }^{3} \longrightarrow \mathrm{P} 680^{1}+\mathrm{O}_{2}{ }^{1}
$$

Although the formation of $\mathrm{P} 680^{3}$ is relatively rare due to fast electron transfer processes, it does occur and

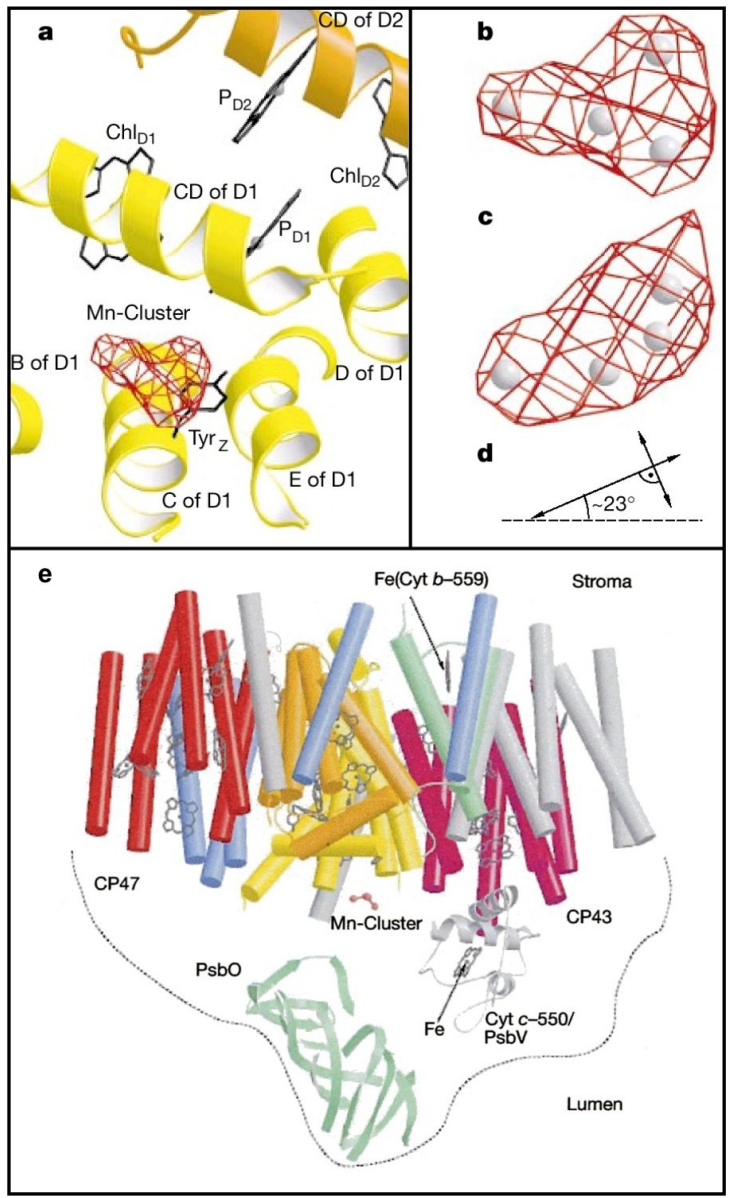

Figure 6. Location and orientation of the $(\mathrm{Mn})_{4}$ cluster taken from Zouni et al. [10]. (a) Close-up view of the reaction centre with the electron density of the $(\mathrm{Mn})_{4}$ cluster. The view is from the lumenal side onto the membrane plane and shows that the $(\mathrm{Mn})_{4}$ cluster is located at the lumenal ends of the $\mathrm{C}$ and $\mathrm{D}$ helices of the $\mathrm{D} 1$ protein, close to the surface $\mathrm{CD}$ helix as predicted by site-directed mutagenesis [14]. (b) Enlarged view of the electron density of the manganese cluster. (c) $90^{\circ}$ rotated around the horizontal axis (view along the membrane with the lumenal side on top). (d) Orientation of the short and long axes of (c). The latter is tilted $23^{\circ}$ against the lumenal side of the membrane plane (hatched line). (e) Side view of the PSII monomer looking down the long axis of the D1/D2 proteins with a slightly tilted angle of the membrane plane so as to show the lumenal surface and the positioning of the $(\mathrm{Mn})_{4}$ cluster, the PsbO protein assigned to the $\beta$-sheet structure (green) and cyt c550 (PsbV). The cylinders show the transmembrane helices of the various subunits within the monomer and can be compared with the lumenal view shown in Figure $4 B$ (note that the colour coding is slightly different). (The figure is taken from Zouni et al. [10] and modified by labelling.)

therefore PSII is vulnerable to $\mathrm{O}_{2}{ }^{1}$ damage. This manifests itself in the selective oxidation of the amino acids 


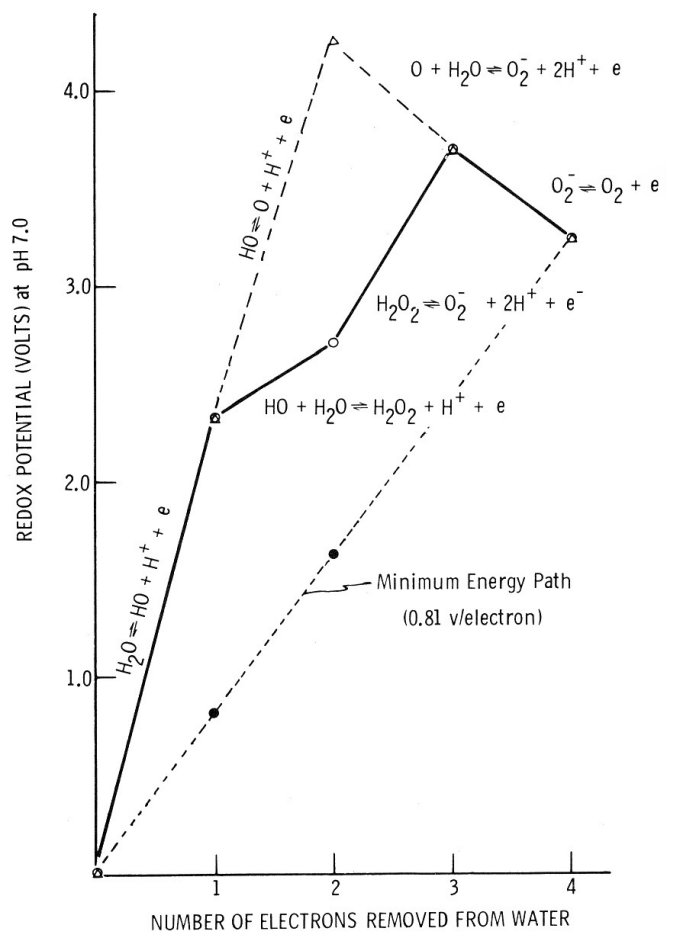

Figure 7. Diagram showing the redox potential of the oneelectron steps for the oxidation of $\mathrm{H}_{2} \mathrm{O}$ to $\mathrm{O}_{2}$. Solid line: pathway via $\mathrm{H}_{2} \mathrm{O}_{2}$ as the two-equivalent reduction stage. Solid and dashed lines: pathway via " $\mathrm{H}_{2} \mathrm{O}+\mathrm{O}$ " as the twoequivalent reduction stage. Dotted line: minimum energy pathway. Taken from Radmer and Cheniae [48].

of the D1 protein [34] and leads to "acceptor side photoinhibition" [31]. The resulting damage of the D1 protein by either process means that it is regularly (every 30 to 60 minutes) degraded and replaced in an illuminated plant or other types of photosynthetic organism.

This remarkable "damage-repair" cycle does not usually involve any other PSII proteins and is therefore highly regulated so as to cause minimal disruption to the PSII complex. Nevertheless, it does require reinsertion of active redox cofactors and the assembly of a functional $4 \mathrm{Mn}$-atom cluster. There are intense studies being carried out to understand how this is achieved and again the recent structural work is helping to formulate a framework on which to interpret results emerging from molecular genetics [35, 36] and biochemistry [37, 38].

The turnover of the D1 protein is part and parcel of the water oxidation properties of PSII and to my mind to understand the mechanisms involved in this amazing phenomenon is as important as unraveling the reactions of water splitting. But what is clear is that oxygenic photosynthetic organisms have developed a range of mechanisms to protect themselves from photodamage, ranging from long term adaptation (regula- tion of the size of the LH systems, cuticle waxes to reflect light, additional non-photosynthetic pigmentation such as astaxanthins) and short term adaptation (leaf curling, chloroplast rotation, generation of quenching centres for excess excitation energy in LH systems, secondary electron donation to $\mathrm{P} 680^{\bullet+}$ from Cyt b559 and $\beta$-carotene within the RC).

\section{THE BIGGER PICTURE}

The PSII RC core binds 33 chlorophyll $a$ molecules, 27 are bound to CP43 and CP47, 4 form P680 and 2 others bind symmetrically to D1 and D2 protein at His 118; $\mathrm{Chl}_{Z-D 1}$ and $\mathrm{Chl}_{\text {Z-D2 }}$ (see Figure 5). These two additional chlorophylls may act as conduits for energy transfer from the CP43 and CP47 chlorophylls. However, as explained above, the cross-section for light absorption by PSII is greatly increased by the association of LH systems with the RC core. The chlorophyll $a / b$ binding proteins of plants and green algal LH systems are encoded by cab genes. Those associated with PSII are known as lhcb proteins, which collectively form the LHCII, CP29, CP26, and CP24 complexes [39]. According to electron crystallography, these proteins have three transmembrane helices and in the case of LHCII associate together to form a trimer [40]. The number of chlorophyll $a$ /chlorophyll $b$ molecules varies between different types of lhcb proteins. In red algae and cyanobacteria, the water soluble phycobiliproteins aggregate to form a large extrinsically located structure known as a phycobilisome [41]. The rods of the phycobilisome are composed of phycobiliproteins such as phycoerythrin and phycocyanin and act like "optical fibres" transferring energy to the chlorophylls of the PSI RC core via a phycobiliprotein core consisting of allophycocyanin.

In all cases, the delivery of excitations from the $\mathrm{LH}$ systems to the PSII RC core is highly efficient. The transfer process seems to be predominantly by Forster's induction resonance mechanism. It is a challenge to understand how these LH systems are structurally coupled to the PSII RC core. In the case of lhcb, considerable progress has been made and the 3D structure of a LHCII-PSII supercomplex has been determined using electron cryoelectron microscopy [42]. This large structure is dimeric and each PSII RC core monomer has associated with it one LHCII trimer and single copies of CP29 and CP26. It therefore binds approximately 100 chlorophyll molecules of which approximately 25 are chlorophyll $b$. At $17 \AA$ resolution the supercomplex structure is providing an excellent framework for the insertion of high resolution structures of PSII RC core and LHCII, as shown in Figure 8, and for understanding the organisation of the extrinsic PsbO, PsbP and PsbQ proteins [43].

Although the structures of many phycobiliproteins have been determined to high resolution, even to $1.4 \AA$ 


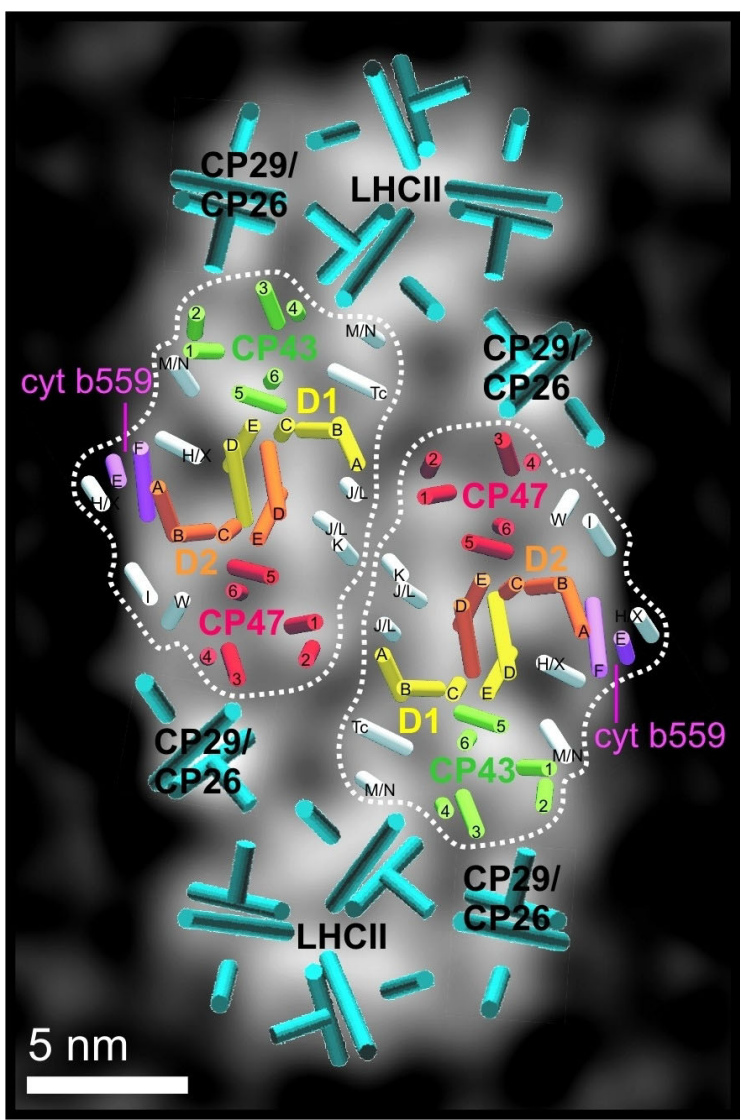

Figure 8. The relationship between the transmembrane helices of the central PSII core dimer and those of the outer antenna system of the LHCII-PSII supercomplex of spinach based on a combination of electron crystallographic and single particle analyses [9, 42]. Helices of the spinach core dimer from Figure 4 and the antenna Cab proteins, Lhcb 1, 2, 4 and 5, based on the structure of LHCII [40] have been incorporated into the 3D map. For the helices of the core dimer, the colour coding and labelling is the same as in Figures 3 and 4, while the modelled helices for LHCII, CP29 and CP26 (including one surface helix) are in cyan.

(e.g., [44]) it is not yet possible to construct a detailed model for their interactions within the phycobilisome. This is because their specific interactions are governed by non-pigmented linker proteins which are required to stack phycobilisome hexamers to form rods [41]. Similarly it is not known how the phycobilisome interacts, via the allophycocyanin core and its anchor protein, with the stromal surface of the PSII RC core.

\section{FINAL COMMENT}

The challenge that faces PSII research is to reveal the structure of the PSII RC core with and without its LH systems attached, at the highest possible spatial resolution. In this way the molecular basis of light intercep- tion, excitation transfer and water oxidation will be better understood. The task is made more complicated by the dynamic nature of PSII, both in terms of the S-state reactions of water oxidation and the "damage-repair" cycle manifested by the turnover of the D1 protein.

Biology found the perfect solution to solve its energy problem but to do so it invented a self-repairing system able to cope with thermodynamically difficult and toxic chemistry. To mimic this system is a challenge and will require more robust components than Nature was forced to use during the evolution of PSII. In this context interesting photoactive $\mathrm{Mn}-\mathrm{Ru}$ coupled systems are being synthesised and studied [45].

\section{ACKNOWLEDGMENT}

I wish to thank the Biotechnology and Biological Research Council for financial support.

\section{REFERENCES}

[1] H. V. Sverdrup, M. W. Johnson, and R. H. Fleming, The Oceans. Their Physics, Chemistry and General Biology. Pubs. Prentice-Hall, Englewood Cliffs, NJ, USA, 1942.

[2] M. D. Archer and J. Barber, Photosynthesis and Photoconversion, Pub. Imperial College Press, London, UK, 2004, pp. 1.

[3] M. Mimuro, K. Hirayama, K. Uezono, H. Miyashita, and S. Miyachi, Biochim. Biophys. Acta 1456 (2000), 27.

[4] D. R. Klug, J. B. Durrant, and J. Barber, Phil. Trans. R. Soc. Lond. A 356 (1998), 449.

[5] D. A. Diner and F. Rappaport, Ann. Rev. Plant Biol. 53 (2002), 551.

[6] K.-H. Rhee, E. P. Morris, D. Zheleva, B. Hankamer, W. Kühlbrandt, and J. Barber, Nature 389 (1997), 522.

[7] K.-H. Rhee, E. P. Morris, J. Barber, and W. Kühlbrandt, Nature 396 (1998), 283.

[8] B. Hankamer, E. P. Morris, and J. Barber, Nature Struct. Biol. 6 (1999), 560.

[9] B. Hankamer, E. P. Morris, J. Nield, C. Gerle, and J. Barber, J. Struct. Biol. 135 (2001), 262.

[10] A. Zouni, H. T. Witt, J. Kern, P. Fromme, N. Krauss, W. Saenger, and P. Orth, Nature 409 (2001), 739.

[11] N. Kamiya and J. R. Shen, Proc. Natl. Acad. Sci. USA 100 (2003), 98.

[12] J. Barber and M. D. Archer, J. Photochem. Photobiol. 142 (2001), 97.

[13] J. Barber, Bioelectrochem. Bioeng. 55 (2002), 135.

[14] B. A. Diner, E. Schlodder, P. J. Nixon, W. J. Coleman, F. Rappaport, J. Lavergne, W. F. J. Vermaas, and D. A. Chisholm, Biochemistry 40 (2001), 9265.

[15] C. W. Hoganson and G. T. Babcock, Science 277 (1997), 199. 
[16] H. Michel and J. Deisenhoffer, Biochem. 27 (1988), 1.

[17] R. J. Debus, Biochim. Biophys. Acta 1102 (1992), 269.

[18] R. J. Debus, Biochim. Biophys. Acta 1503 (2001), 164.

[19] C. Tommos and G. T. Babcock, Biochim. Biophys. Acta 1458 (2000), 199.

[20] C. Tommos, Phil. Trans. R. Soc. Lond. 357 (2002), 1383.

[21] J. S. Vrettos, J. Limburg, and G. W. Brudvig, Biochim. Biohys. Acta 1505 (2001), 229.

[22] J. S. Vrettos and G. W. Brudvig, Phil. Trans. R. Soc. Lond. 357 (2002), 1395.

[23] M. Hanmann and W. Junge, Biochim. Biophys. Acta 411 (1999), 8189.

[24] W. Junge, M. Hanmann, R. Ahlbrink, A. Mulkidjanian, and J. Clausen, Phil. Trans. R. Soc. Lond. 357 (2002), 1407.

[25] B. A. Diner, Biochim. Biophys. Acta 1503 (2001), 147.

[26] T. M. Bricker and L. K. Frankel, Photosynth. Res. 56 (1998), 157.

[27] T. G. Carrell, A. M. Tyryshkin, and G. C. Dimusktes, J. Biol. Inorg. Chem. 7 (2001), 2.

[28] J. M. Peloquin and R. D. Britt, Biochim. Biophys. Acta 1503 (2001), 96.

[29] V. K. Yachandra, Phil. Trans. R. Soc. Lond. 357 (2002), 1347.

[30] J. Nugent, Biochim. Biophys. Acta 1503 (2001), 1.

[31] J. Barber and B. Andersson, Trends Biochem. Sci. 17 (1992), 61.

[32] J. R. Durrant, L. B. Giorgi, J. Barber, D. R. Klug, and G. Porter, Biochim. Biophys. Acta 1017 (1990), 141.

[33] A. Telfer, S. Dhami, S. M. Bishop, D. Phillips, and J. Barber, Biochem. 33 (1994), 14469.
[34] J. Sharma, M. Panico, C. A. Shipton, F. Nilsson, H. R. Morris, and J. Barber, J. Biol. Chem. 272 (1997), 33158.

[35] E. Baena-Gonzalez and E.-M. Aro, Phil. Trans. R. Soc. Lond. 357 (2002), 1451.

[36] P. S. Silva, Y.-J. Choi, H. A. G. Hassan, and P. J. Nixon, Phil. Trans. R. Soc. Lond. 357 (2002), 1461.

[37] M. Lindahl, C. Spetea, T. Hundal, A. B. Openheim, Z. Adam, and B. Andersson, Plant Cell 12 (2000), 419.

[38] K. Haußühl, B. Andersson, and I. Adamska, EMBO J. 20 (2001), 713.

[39] S. Jansson, Biochim. Biohys. Acta 1184 (1994), 1.

[40] W. Kühlbrandt, D. N. Wang, and Y. Fujiyoshi, Nature 367 (1994), 614.

[41] A. N. Glazer, Ann. Rev. Microbiol. 36 (1982), 173.

[42] J. Nield, E. V. Orolva, E. P. Morris, B. Gowen, M. van Heel, and J. Barber, Nature Struct. Biol. 7 (2000), 44.

[43] J. Nield, M. Balsera, J. De Las Rivas, and J. Barber, J. Biol. Chem. 277 (2002), 15006.

[44] J. Nield, P. J. Rizkallah, J. Barber, and N. E. Chayen, J. Struct. Biol. 141 (2003), 149.

[45] P. Huang Kenez, A. Magnuson, R. Lomoth, M. Abrahamsson, M. Tamm, L. Sun, B. van Rotterdam, J. Park, L. Hammarström, B. Åkermark, and S. Styring, J. Inorg. Biochem. 91 (2002), 159.

[46] B. Kok, B. Forbush, and M. McGloui, Photochem. Photobiol. 11 (1970), 457.

[47] B. Hankamer, E. P. Morris, J. Nield, A. Carne, and J. Barber, FEBS Lett. 504 (2001), 142.

[48] R. Radmer and G. Cheniae, In Topics in Photosynthesis. Vol. 2 (J. Barber, Ed.), Pub. Elsevier, Amsterdam, 1977, pp. 303. 


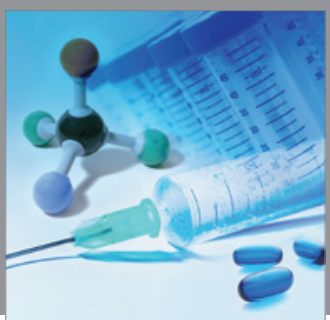

International Journal of

Medicinal Chemistry

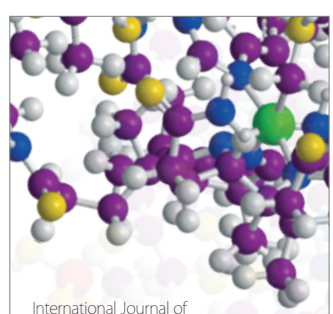

Carbohydrate Chemistry

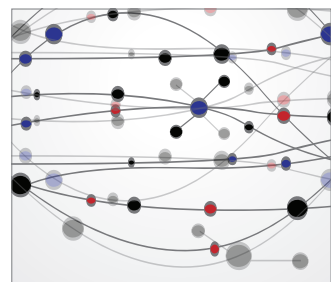

The Scientific World Journal
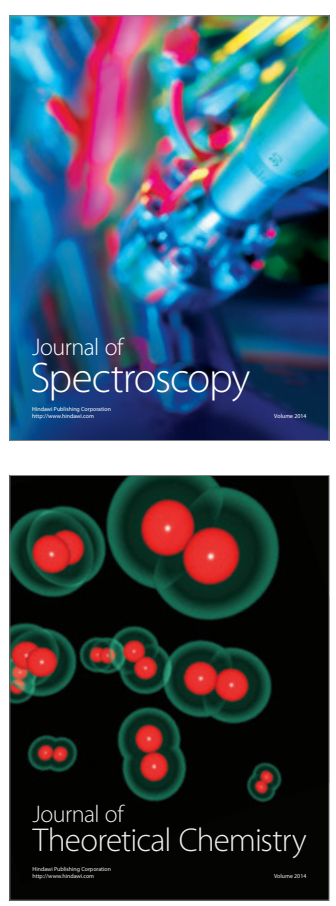
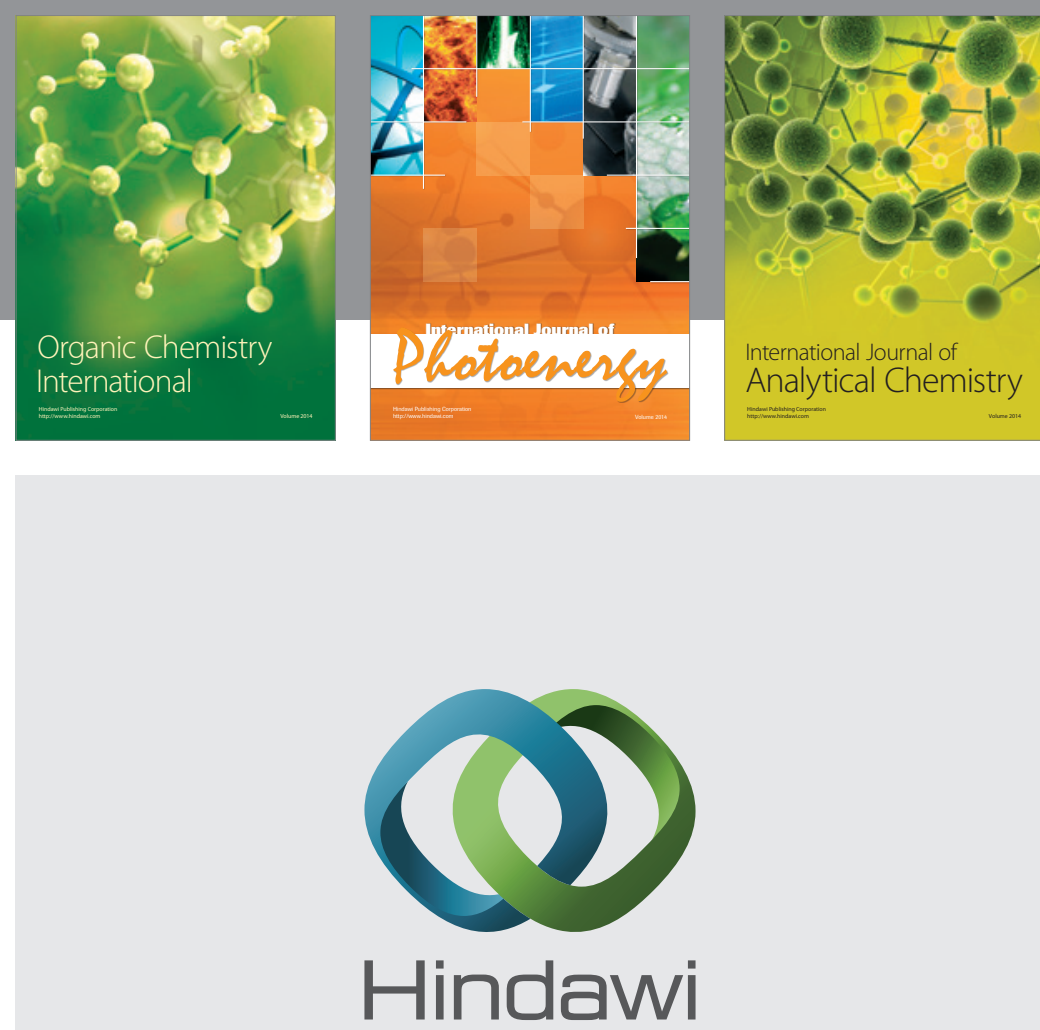

Submit your manuscripts at

http://www.hindawi.com
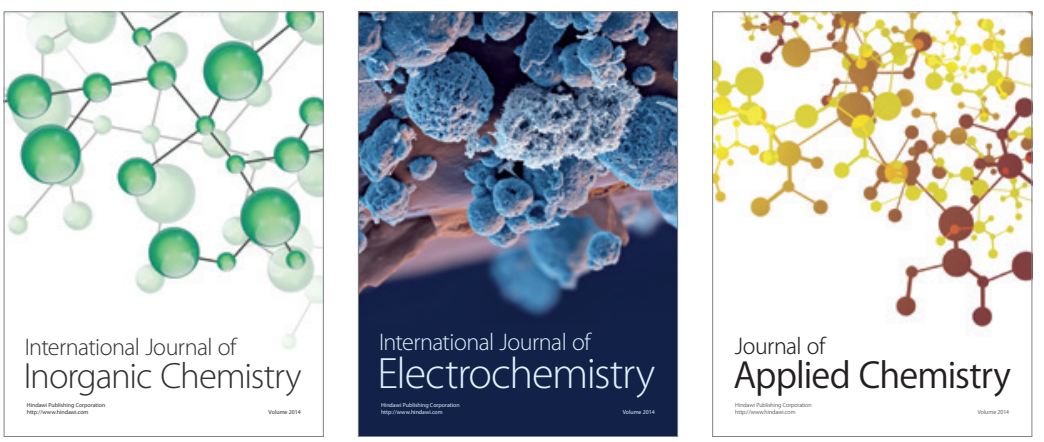

Journal of

Applied Chemistry
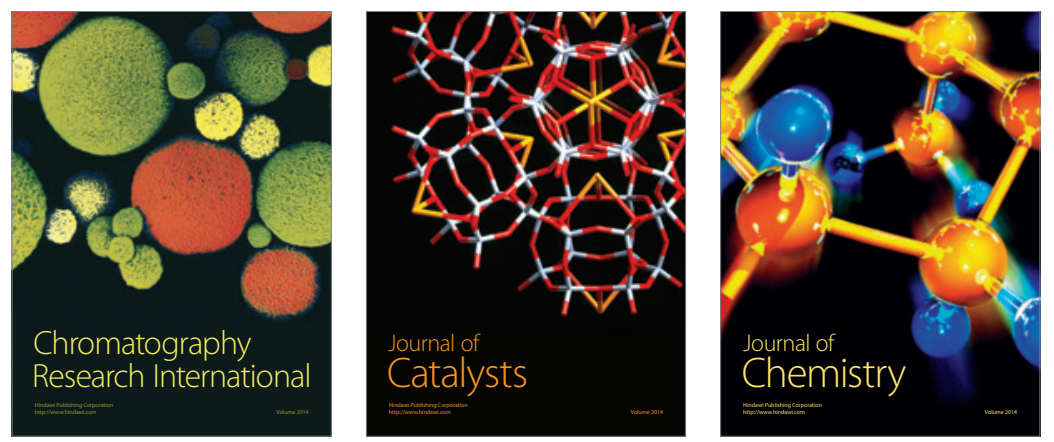
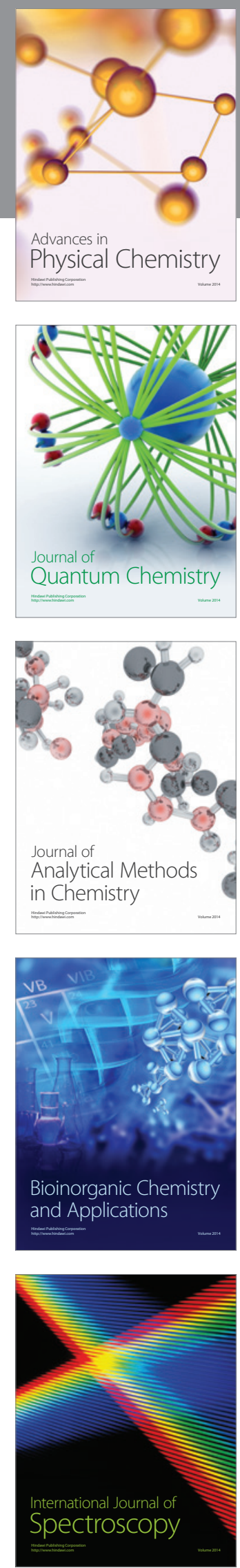